\title{
Plasma Calcium, Phosphorous and Magnesium Levels in Cats with and without Tooth Resorption
}

\section{Diana L Eubanks}

Associate Clinical Professor, Department of Clinical Sciences, Mississippi State University, Mississippi State, USA

\begin{abstract}
Feline tooth resorption is a common and painful condition affecting many middle-aged and older cats. Though many theories exist on the cause of this condition, no etiology has yet been determined. It is likely that multiple factors are involved in the development and progression of tooth resorption including cytokines, growth factors, and plasma and crevicular fluid levels of key electrolytes, tissue $\mathrm{pH}$, and activators of osteoclasts. This study examined 40 client-owned cats for the presence of tooth resorption. Serum levels of calcium, phosphorous, and magnesium were measured as well as the $\mathrm{pH}$ of oral tissues. Results did not demonstrate an association between electrolyte levels or $\mathrm{pH}$ and the presence of resorptive lesions. The overall prevalence of tooth resorption was lower than that of most previously published studies.
\end{abstract}

Keywords: Feline; Tooth; Resorption; Osteoclasts; Calcium; Phosphorous; Magnesium

Abbreviations: TR: Tooth Resorption; PDL: Periodontal Ligament

\section{Introduction}

Feline tooth resorption (formerly known as Feline Odontoclastic Resorptive Lesions) affects between 28.5 and 67 percent of middle aged domestic cats [1,2].This common disease process of the tooth substance causes extreme discomfort for the cat, resulting in vocalization and dropping of food, especially as the pulp is exposed by progression of the disease.

The etiology of the inciting causes and the factors contributing to the progression of tooth resorption (TR) have not been determined although many theories regarding causation exist.Over the past five decades, the incidence of TR has increased, and it has been speculated that this increase may be associated with aspects of domestication such as diet, vaccinations, and environment [3]. Experts agree that osteoclasts are the primary cell involved in the degradation of tooth substance resulting in resorptive lesions [4].

Whereas some TR can be identified on gross oral examination, many additional lesions can be detected utilizing intra-oral radiology. In a study by [5] a rapid screening test for overall tooth resorption using two radiographic views (bilateral mandibular premolars and molars) correctly predicted the overall presence or absence of TR in 93.4 percent of the animals studied [5].

In addition to TR, a variety of other musculo-skeletal diseases (hypervitaminosis A, nutritional and secondary hyperparathyroidism, arthritis, neoplasia and non-union fractures) involving increased bone resorption by osteoclasts affect aging cats [6].Currently, there is little information available concerning the biology of osteoclasts in the feline [4].

In a recent study, evaluation of grossly normal teeth from cats with resorptive lesions in other areas of the mouth revealed degeneration of the periodontal ligament(PDL) and narrowing of the PDL space, hyperemia, edema, hyperosteoidosis, as well as areas of ankylosis, supporting the theory that early TR is a non-inflammatory replacement resorption that occurs independent of periodontal disease [1].In humans, external root resorption is classified as surface, replacement, or inflammatory with surface resorption being self- limiting and replacement resorption resulting in ankylosis of the tooth, a complication commonly associated with the reimplantation of teeth [1]. All cats suffer from some degree of surface cementum resorption; however, areas of TR develop in cats when these so-called "normal" lesions fail to heal. Cats that do not develop lesions may have more effective healing. It has been speculated that a cat's healing ability may decline with age, making it inversely proportional to its chances of developing TR.

A better understanding of this perceived impairment of healing ability in the teeth of older cats may provide the key to understanding the cause of feline tooth resorption. Calcium, phosphorous and magnesium are key ingredients in the composition of teeth. Their bioavailability is important in the maintenance and repair of resorptive lesions. Resorption and repair are cyclic in nature. Deficiencies in serum electrolytes such as calcium, phosphorous, and magnesium may perhaps contribute to the inability of some cats to shift the resorption/ repair cycle towards repair. Influence from other chemical mediators in the body such as cytokines, hormones, and inflammatory mediators may also be significant.

In this study, we measured the plasma concentrations of three electrolytes, calcium, phosphorous and magnesium, in 40 middle-aged, client-owned cats and also examined the cats for the presence of tooth resorption.

\section{Materials and Methods}

Forty client-owned cats, between the ages of 4 and 8 years, were presented to the Mississippi State College of Veterinary Medicine

Corresponding author: Diana L Eubanks, Associate Clinical Professor Department of Clinical Sciences, Mississippi State University, PO Box 6100 Mississippi State, MS 39762, USA, Tel: 662-325-1435; E-mail: deubanks@cvm. msstate.edu

Received August 12, 2011; Accepted October 11, 2011; Published October 16 2011

Citation: Eubanks DL (2011) Plasma Calcium, Phosphorous and Magnesium Levels in Cats with and without Tooth Resorption. J Veterinar Sci Technol S3:001. doi:10.4172/2157-7579.S3-001

Copyright: (c) 2011 Eubanks DL. This is an open-access article distributed under the terms of the Creative Commons Attribution License, which permits unrestricted use, distribution, and reproduction in any medium, provided the original author and source are credited. 
Small Animal Dental Service for a routine dental prophylaxis. Cat's ages were based on information provided by the clients. The initial examination included a physical exam, serum chemistry panel, CBC, and a brief oral exam. Cats were housed in individual cages until the scheduled procedure. Anesthetic regimes varied and were chosen based on health status of the patient, perceived level of pain involved with the procedure, and anesthetist preference. The cats were then placed under general anesthesia and positioned on a circulating water heating pad. Monitoring consisted of ECG, blood pressure, heart rate, and oxygen saturation. A full oral exam was performed as well as intra-oral radiographs of teeth number 307-309 and 407-409 (left and right mandibular $3^{\text {rd }}$ and $4^{\text {th }}$ premolars and left and right mandibular molars) using a parallel technique. The oral exam included periodontal grading using a modified Loe and Silness index and measurement of oral $\mathrm{pH}$ using a commercially available $\mathrm{pH}$ strip ${ }^{\mathrm{a}}$. All tooth surfaces were inspected visually, explored using a number 23 shepherd's crook explorer, and probed to assess sulcus depth with an NC periodontal probe. Any abnormal findings were recorded on the patient's dental chart.

Following the dental examination, cats were placed in one of two categories:

\section{Group A: cats having TR}

Group B: cats with no discernable signs of TR based on gross oral examination and intra-oral radiographs of the most commonly affected teeth

Any cat showing evidence of tooth resorption was given additional pain medication and the tooth/teeth was/were treated appropriately. Cats were recovered and returned to their owners. Owners completed a brief survey related to their cats' eating habits and environment.

Statistical analysis was performed using a one-way ANOVA.

\section{Results}

Six of the 40 cats (15\%) showed evidence of tooth resorption, based on oral probing, radiographs or both. There were an equal number of male and female cats with TR and older cats were more likely to be affected. (The average age of cats in the study was $5.5 \mathrm{yr}$ and the average age of cats with TR was $6.7 \mathrm{yr}$ ). One cat with TR was reportedly 4 years of age, one was 5 years and the others were 7 years or greater.

Owners reported that all cats were either strictly indoors or indoor/ outdoor cats and all received dry kibble as their primary diet. One cat with TR also received occasional canned food and one cat without TR received boiled chicken.

There were no significant differences in oral $\mathrm{pH}$ measurements or periodontal index measurements (plaque, calculus, or gingival) between cats with and without TR.

Plasma calcium and magnesium levels were the same in cats with and without TR. Cats with TR were shown to have lower plasma phosphorous levels than cats without TR though the differences were not statistically significant.

\section{Discussion}

A $15 \%$ prevalence of tooth resorption in this study is lower than that reported in other similar studies. Many studies have relied on gross evaluation of the oral cavity, periodontal grading, and intraoral radiographs similar to the measurements performed in this study. Other studies have incorporated more advanced imaging and histological evaluation of tissues which may increase the likelihood of identifying early lesions. The lack of gender predilection as well as the higher incidence of TR in older cats is consistent with previous findings.

Calcium, phosphorous and magnesium are important elements in the maintenance of normal tooth structure. Many alterations in bioavailability of these substances occur between the bloodstream and the tooth itself. Additionally, influence from other chemical mediators in the body such as cytokines, hormones, and inflammatory mediators may alter the action of these substances. Levels of calcium, phosphorous and magnesium in feline saliva as well as gingival crevicular fluid may provide a better indication of the relationship between these elements and the presence or absence of TR in the feline. More advanced methods of detection of tooth resorption may also yield different results and should be considered in future studies. Additional measurements of other biochemical markers may also aid in elucidating the cause of both early lesions and the perpetuating factors that lead to advanced resorptive lesions.

Collection and measurement of saliva and gingival crevicular fluid in the feline are difficult due to the small sampling size. If sufficient samples can be collected, the measurement of electrolytes, cytokines, inflammatory mediators, etc in these fluids may reflect a link between oral fluid components and the presence or absence of tooth resorption.

${ }^{\mathrm{a}} \mathrm{pHion}$ test strip, pHion.com

\section{References}

1. Gorrel C, LarssonA (2002) Feline ondotoclastic resorptive lesions: unveiling the early lesion. J Small Anim Pract 43: 482-488.

2. Verstraete FJM, Van Aarde RJ, Nieuwoudt BA, Mauer E, Kass PH (1996) The dental pathology of feral cats on Marion Island, Part 11: Periodontitis, external odontoclastic resorptive lesions and mandibular thickening. Journal of Comparative Pathology 115: 283-297.

3. Reiter AM, Mendoza KA (2002) Feline ondotoclastic resorptive lesions an unsolved enigma in veterinary dentistry.Vet Clin North Am Small Anim Pract 32: 791-837.

4. Muzylak M, Arnett TR, Price JS, Horton MA (2007) The in vitro effect of pH on osteoclasts and bone resorption in the cat: implications for the pathogenesis of FORL. J Cell Physiol 213: 144-150.

5. Heaton M, Wilkinson J, Gorrel C, Butterwick R (2004) A rapid screening technique for feline odontoclastic resorptive lesions. J Small Anim Pract 45 598-601.

6. Muzylak M, Flanagan AM, Ingham K, Gunn N, Price J, et al. (2002) A feline assay using osteoclasts generated in vitro from peripheral blood for screening anti-resorptive agents. Res Vet Sci 73: 283-290. 